\title{
Verbal memory and hippocampal volume predict subsequent fornix microstructure in those at risk for Alzheimer's disease
}

\author{
Junhong $\mathrm{Yu}^{1,2}$ - Tatia M. C. Lee $\mathrm{e}^{1,2,3,4} \cdot$ for the Alzheimer's Disease Neuroimaging Initiative
}

Published online: 7 September 2019

(C) The Author(s) 2019

\begin{abstract}
While strong cross-sectional evidence supported the use of fornix microstructure as a marker for detecting Alzheimer's disease (AD), longitudinal data remains inconclusive on the sequential nature of fornix microstructure abnormalities and AD progression. An unequivocal longitudinal relationship between fornix microstructure and markers of AD progression -memory impairment and hippocampal atrophy, must be established to validate fornix microstructure as a marker of AD progression. We included 115 participants from the Alzheimer's Disease Neuroimaging Initiative across the non-demented AD spectrum — defined as those who had at least one $\mathrm{AD}$ risk marker at baseline (e.g., mild cognitive impairment (MCI) due to AD diagnosis, amyloid or ApoE4 positivity) and/or 'cognitively normal individuals who converted to $\mathrm{MCI}$ due to $\mathrm{AD}$ or $\mathrm{AD}$, with structural and diffusion tensor imaging scans at baseline and two years follow-up. Hippocampal volumes (HV), fractional anisotropy (FA) and mean diffusivity (MD) in the fornix were extracted. Memory was indexed via composite scores of verbal memory tests. Structural equation models tested the bidirectional cross-lagged effects of fornix microstructure, memory, and HV. Impaired memory and smaller HV at baseline significantly predicted worse fornix microstructure (decreased FA and increased MD) two years later. Baseline fornix microstructure was not associated with subsequent changes in memory and HV. Fornix microstructure is compromised likely at a later stage, where significant decline in memory and hippocampal atrophy have occurred. This limits the utility of fornix microstructure in the early detection of AD. Our findings inform the possible pathophysiology and refined the use of AD neural markers.
\end{abstract}

Keywords Alzheimer’s disease · ADNI · Fornix $\cdot$ Hippocampus $\cdot$ Memory $\cdot$ Longitudinal

Data used in preparation of this article were obtained from the Alzheimer's Disease Neuroimaging Initiative (ADNI) database (adni.loni.usc.edu). As such, the investigators within the ADNI contributed to the design and implementation of ADNI and/or provided data but did not participate in analysis or writing of this report. A complete listing of ADNI investigators can be found at: http://adni.loni.usc.edu/wpcontent/uploads/how_to_apply/ADNI_Acknowledgement_List.pdf.

Electronic supplementary material The online version of this article (https://doi.org/10.1007/s11682-019-00183-8) contains supplementary material, which is available to authorized users.

Tatia M. C. Lee

tmclee@hku.hk

1 The State Key Laboratory of Brain and Cognitive Sciences, The University of Hong Kong, Pokfulam, Hong Kong

2 Laboratory of Neuropsychology, The University of Hong Kong, Rm 656, Jockey Club Tower, Pokfulam Road, Hong Kong, Hong Kong

3 Institute of Clinical Neuropsychology, The University of Hong Kong, Pokfulam, Hong Kong

4 Guangdong-Hong Kong-Macao Greater Bay Area Center for Brain Science and Brain-Inspired Intelligence, Guangzhou, China

\section{Introduction}

Alzheimer's disease (AD) is a chronic neurodegenerative condition characterized by progressive memory impairment. Early detection and intervention are crucial to maximize prognostic outcomes. To this end, non-invasive neural markers have been suggested to facilitate early detection (Sperling et al. 2011). One candidate marker that has garnered recent interest is the white matter (WM) microstructure of the fornix, which can be observed via the diffusion tensor imaging (DTI) indices of fractional anisotropy (FA) and mean diffusivity (MD). The fornix is a bundle of WM fibers that connects the hippocampus to subcortical structures. Due to this structural association with the hippocampus - a region that is crucially implicated in both short-term and long-term memory, it has been widely studied in AD. Cross-sectional studies using DTI have revealed significantly altered WM microstructure in the fornix as indicated by decreased FA and increased 
MD, among individuals with $\mathrm{AD}$ (Oishi and Lyketsos 2014), as well as its prodromal amnestic mild cognitive impairment (aMCI) stage ( $\mathrm{Yu}$ et al. 2017). Whilst these findings provide strong preliminary evidence, their crosssectional nature limits their ability to illustrate the sequential nature of the relationship between progressive memory decline and changes in fornix microstructure, which would be an important consideration in using fornix DTI measures as a marker of $\mathrm{AD}$ progression.

The limited longitudinal findings on fornix microstructure and memory impairment are however, less consistent and have failed to arrive at a satisfactory conclusion regarding the precise nature of the relationship between fornix microstructure and memory impairment. A few studies have looked at how baseline fornix WM indices predict subsequent memory outcomes. Mielke et al. (2012) found fornix FA, but not $\mathrm{MD}$, predicted subsequent memory decline across a 2.5 year period among participants with aMCI. In a study of healthy participants who converted to aMCI two years later (Zhuang et al. 2012), these converters had significantly reduced fornix FA at baseline. Fletcher et al. (2013) on the other hand, found neither baseline fornix FA or MD among healthy participants predicted their subsequent conversion to mild cognitive impairment (MCI; unclear if they were of the amnestic subtype) or AD. Nevertheless, they found baseline fornix axial diffusivity, but not radial diffusivity, to be related to subsequent conversion to $\mathrm{MCI}$ or $\mathrm{AD}$. Two other studies examined the reverse association (i.e., how memory impairment at baseline predicted subsequent changes in fornix WM indices). Teipel et al. (2010) compared changes in fornix FA across a 13-to-16 months period among participants identified as aMCI and healthy controls at baseline. They noted that there was a general decrease in fornix FA across the entire studied sample. However, there was not a significant time by group interaction effect to suggest that the decline in fornix FA is specific to aMCI. In another study, fornix MD increased significantly over a 1-year period among participants with aMCI, relative to healthy controls. Interestingly, such differences between participants with $\mathrm{AD}$ and healthy controls were not significant despite significant baseline differences. Furthermore, none of the between-group differences in longitudinal FA changes were statistically significant (Nowrangi et al. 2013).

These longitudinal studies were subject to some methodological limitations. Firstly, the majority of these studies (Fletcher et al. 2013; Nowrangi et al. 2013; Teipel et al. 2010; Zhuang et al. 2012) analyzed binary memory-related diagnostic outcomes, instead of memory test scores on a continuum. While they intended to study these diagnoses rather than memory per se, this binary approach may not adequately capture the variability in memory impairment across the $\mathrm{AD}$ spectrum, and thus may risk oversimplifying the relationship between memory impairment and fornix WM changes.
Understandably, the small sample sizes of these studies may not make it practical to operationalize memory impairment on a continuum of test scores. Secondly, studies (Fletcher et al. 2013; Mielke et al. 2012; Zhuang et al. 2012) that examined how baseline fornix WM indices may predict subsequent memory-related outcomes did not control for baseline levels of memory impairment. It is possible that those participants with worse fornix microstructure relative to others at baseline may also start off with worse memory impairment. As a result, they will naturally present with worse memory impairment at follow-up, simply because of their lower baseline levels.

If such disruptions in fornix microstructure do indeed occur in the context of AD-related memory impairment, there may be two possible explanations. According to the conventional amyloid cascade hypothesis, memory impairment in AD has been theorized to be a result of amyloid-beta mediated neuronal death (Hardy and Selkoe 2002). Kantarci (2014) suggested that the death of hippocampal neurons would subsequently lead to the degeneration of the fornix axons, especially since most of the axonal projections carried by the fornix originate from the hippocampus. Alternatively, memory loss may not always be a result of neuronal loss (Caso et al. 2016); a more recent theory implicates immune-system mediated neuroinflammatory processes in the pathogenesis of $\mathrm{AD}$ (Heppner et al. 2015). Neuroinflammation observable via WM microstructural changes may also contribute to memory impairment (Caso et al. 2016). There is little experimental or longitudinal evidence to support either explanation. Unraveling the longitudinal relationship between hippocampal atrophy and fornix microstructure would be a useful first step in evaluating these theories.

In light of the above gaps in knowledge, the current study examined the longitudinal relationship between fornix microstructure and other non-invasive markers of $\mathrm{AD}$ progression such as verbal memory and hippocampal volume. The aim was to examine the antecedents and consequences of such fornix microstructural changes in the onset of AD. This information will be highly relevant in evaluating the validity of using fornix microstructure in the early detection of $\mathrm{AD}$. Additionally, these findings are important to understand the pathophysiological mechanisms involved in the prodromal stages of the disease. Using the longitudinal data from the Alzheimer's Disease Neuroimaging Initiative (ADNI), we examined auto-regressive cross-lagged models of fornix microstructure and memory impairment, as well as that of fornix microstructure and hippocampal atrophy. In the former, in line with the reviewed evidence, we hypothesized a bidirectional relationship. Specifically, worse fornix microstructure, observed via decreased FA and increased MD, would be associated with subsequent verbal memory impairments and greater verbal memory impairments would predict worse fornix microstructure subsequently. As for the latter model, we hypothesized that smaller hippocampal volumes at baseline would 
predict worse fornix microstructure at follow-up, in line with Kantarci's (2014) explanations.

\section{Materials and methods}

Data used in the preparation of this article were obtained from the ADNI database (adni.loni.usc.edu). The ADNI was launched in 2003 as a public-private partnership, led by Principal Investigator Michael W. Weiner, MD. The primary goal of ADNI has been to test whether serial magnetic resonance imaging (MRI), positron emission tomography (PET), other biological markers, and clinical and neuropsychological assessments can be combined to measure the progression of MCI and early AD. Ethical approval for the ADNI study was obtained by the ADNI investigators. For up-to-date information, see www.adni-info.org.

\section{Participants}

Participants on the ADNI database with valid structural T1 and/or DTI scans at the baseline and two-year follow-up, specifically from the ADNI GO and ADNI 2 phases, were potential subjects of this study. Informed consent was obtained from all individual participants included in the study. Given that cross-lagged analyses depends not just on within subject variance, but that of between subject as well, it is important to capture as much variance as posssible across the heterogenous spectrum AD spectrum. Nevertheless, if the sample is too heterogenous, the interpretation and generalization of the results would be difficult. Hence there is a need to balance between maximizing variance across the included sample and also interpretability of the results, as such we limited the heterogeneity of the participants by excluding participants at both ends of the AD spectrum - cognitive normal participants without any $\mathrm{AD}$ related biomarkers and those who were already diagnosed with AD. Furthermore, the exclusions of the former group is further justified to enable the findings of the current study to be as specific to $\mathrm{AD}$ as possible and the exclusion of the latter group is further justified by the fact that we are primarily interested in studying the early phases of AD and not individuals who are already afflicted with AD. To these ends we restricted the participants' inclusion to those of the nondemented AD spectrum as defined by one or more of the following criteria:

a) Being diagnosed with $\mathrm{MCI}$ due to $\mathrm{AD}$ at baseline

b) Cognitively normal participants at baseline who converted to probable $\mathrm{AD}$ or $\mathrm{MCI}$ due to $\mathrm{AD}$ at subsequent time points (including those beyond the follow-up if such data is available in the ADNI dataset) c) Cerebrospinal fluid (CSF) amyloid-beta positive $(\mathrm{A} \beta+)$, as defined by having CSF amyloid-beta 1 to 42 peptide $\left(\mathrm{A} \beta_{1-42}\right)$ concentration levels $\leq 192 \mathrm{pg} / \mathrm{mL}$

d) Having at least a single copy of the Apolipoprotein $\varepsilon 4$ gene (APOع4+).

The diagnosis of MCI due to AD was made by a physician during the participant's in-clinic visit. Briefly, the MCI diagnosis has to fulfill all of the following criteria: presence of a subjective memory concern, scores of Logical Memory II from the Wechsler Memory Scale below education-adjusted cutoffs, Mini-mental Status Examination (MMSE) scores above 23 and Clinical Dementia Rating (CDR) of 0.5 . Subsequently, the suspected cause of the MCI was determined to be due to $\mathrm{AD}$ after ruling out all other possible etiologies. Using the same tests, the diagnosis of probable AD was made according to the National Institute of Neurological and Communication Disorders/Alzheimer's Disease and Related Disorders Association criteria. This criteria for AD was similar to MCI due to $\mathrm{AD}$ in terms of requiring a subjective memory concern, Logical Memory II scores below similar cutoffs and the ruling out of all other possible etiologies. On top of that, the participant is required to have an MMSE score between (inclusive) 20 to 26 and a CDR of 0.5 or 1.0. Detailed cut-off scores of the different tests and other criteria for these diagnoses were reported in the ADNI procedures manual (https://adni.loni.usc.edu/wp-content/uploads/2008/07/adni2procedures-manual.pdf). The collection, processing, and storage of CSF were carried out according to the ADNI procedures manual; CSF samples were acquired at the ADNI clinical centers, frozen at $-80^{\circ} \mathrm{c}$ and shipped for analysis to the ADNI Biomarker Core. CSF A $\beta_{1-42}$ concentration values were obtained from analyzing these samples using the xMAP Luminex platform and Fujirebio AlzBio3 immunoassay kit at the UPenn/ADNI Biomarker Laboratory, according to the kit's manufacturer instructions. The cutoff of CSF $\mathrm{A} \beta_{1-42} \leq 192 \mathrm{pg} / \mathrm{mL}$ had high sensitivity (96\%) and specificity (77\%) in classifying AD and cognitively normal cases in an ADNI sample (Shaw et al. 2009).

Next, we further removed three participants due to image quality issues. The remaining 115 participants (54 females) were included in this study. The number of participant with the various AD-spectrum characteristics are presented in a venn diagram in Fig. 1. Among the 115 participants, 98 and 112 participants had valid DTI and T1 structural scans, respectively, at both time points. The characteristics of these participants are presented in Table 1.

\section{Memory scores}

We used the ADNI-MEM score (Crane et al. 2012) - a composite measure of multiple verbal memory tests to 
Fig. 1 Venn diagram showing the included participants with the various AD-spectrum related characteristics. CSF $\mathrm{A} \beta=$ Cerebrospinal fluid amyloid-beta; $\mathrm{AD}=$ Alzheimer's disease $\mathrm{MCI}=$ Mild cognitive impairment

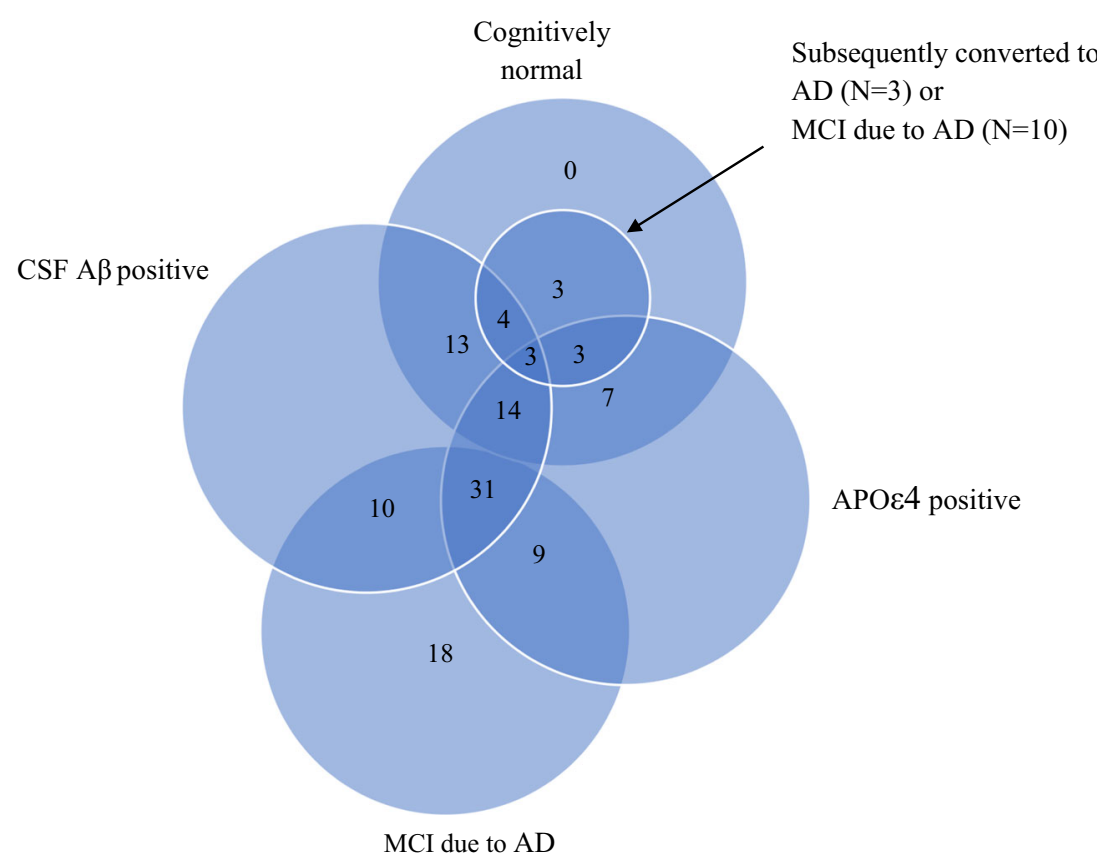

\section{MRI acquisition}

Participants were scanned with General Electric Healthcare $3 \mathrm{~T}$ scanners at various participating sites. T1-weighted images were acquired using a 3D IR-SPGR protocol $(\mathrm{TE}=$ $3.036 \mathrm{~s} ; \mathrm{TR}=7.34 \mathrm{~s} ; \mathrm{TI}=400 \mathrm{~ms} ; 256 \times 256$ matrix; 196
Table 1 Characteristics of included participants

\begin{tabular}{ll}
\hline & Mean (SD)/Frequency (\%) \\
\hline Age $_{\text {baseline }}$ & $73.1(6.9)$ \\
Gender & \\
Male & $61(53.0 \%)$ \\
Female & $54(47.0 \%)$ \\
Years of education & $16.0(2.6)$ \\
Neurocognitive diagnosis & \\
Cognitively normal at baseline & $47(40.9 \%)$ \\
Remained at cognitively normal at follow-up ${ }^{\text {a }}$ & $34(72.3 \%)$ \\
Converted to MCI due to AD at follow-up & $10(21.3 \%)$ \\
Converted to AD at follow-up & $3(6.4 \%)$ \\
MCI due to AD at baseline & $68(59.1 \%)$ \\
Remained at MCI due to AD at follow-up & $52(76.5 \%)$ \\
Converted to AD at follow-up & $16(23.5 \%)$ \\
MMSE scores ${ }_{\text {baseline }}$ & $28.0(1.8)$ \\
Ethnicity & \\
Caucasian & $107(93.0 \%)$ \\
Native American & $1(.9 \%)$ \\
Asian American & $3(2.6 \%)$ \\
African American & $4(3.5 \%)$ \\
\hline
\end{tabular}

$\mathrm{SD}$, standard deviation. $\mathrm{MCI}$ due to $\mathrm{AD}=$ Mild cognitive impairment due to Alzheimer's disease

${ }^{a}$ Among these stable cognitively normal participants, 27 are CSF A $\beta$ positive and 21 are APO $\varepsilon 4$ positive 
sagittal slices; in-plane resolution $=1.0156 \mathrm{~mm}$; slice thickness $=1.2 \mathrm{~mm}$ ). DTI images were acquired with an EPI SE protocol $(\mathrm{TE}=90 \mathrm{~ms} ; \mathrm{TR}=9.05 \mathrm{~s} ; 256 \times 256$ matrix, 59 axial slices, in-plane resolution $=1.37 \mathrm{~mm}$; slice thickness $=$ $2.7 \mathrm{~mm}$; diffusion-weighted volumes in 41 directions; b-val$\mathrm{ue}=1000 \mathrm{~s} / \mathrm{mm}^{2}$ ). Further information regarding these protocols is available online (http://adni.loni.usc.edu/methods/ documents/mri-protocols/).

\section{Image preprocessing}

The raw diffusion images were skull-stripped and corrected for eddy current and subject movement using FMRIB Software Library (FSL 5.0.8). Diffusion tensors were then generated for each participant. We used an unbiased approach (Keihaninejad et al. 2013) for the longitudinal registration of the diffusion tensors. For each subject, a within-subject template was generated by computing the initial average template as a Log-Euclidean mean of the baseline and follow-up diffusion tensors. This template was iteratively refined; the diffusion tensors were registered to the template, and a refined average template was similarly computed from the two registered diffusion tensors for the next iteration. The process was repeated until the difference between templates from consecutive iterations became sufficiently small; first with affine and then with non-linear registrations. Next, a study template was created from all the final average templates in the withinsubject space using the same iterative method. Then, FA and MD maps were generated from each registered tensor image in the study template space. These preprocessing steps were carried out using the diffusion tensor imaging toolkit (DTI-TK). Subsequently, a mean FA skeleton representing the centers of fiber tracts common to all participants, thresholded at FA $>.30$, was created and then projected onto the FA and MD maps prior to the region-of-interests (ROI) extraction.

The structural T1 images were preprocessed longitudinally using the Computational Anatomy Toolbox (CAT12), as implemented in SPM12. Baseline and follow-up T1 images within a subject were rigidly realigned to correct for differences in head position and a subject-specific average template was computed and used as reference in a subsequent realignment of the baseline and follow-up T1 images. The realigned images were segmented into gray matter (GM), WM and CSF, as well as corrected for signal inhomogeneities with reference to the subject average template. These GM and WM images were used to estimate spatial normalization deformation fields using the high dimensional Diffeomorphic Anatomic Registration Through Exponentiated Lie Algebra (DARTEL) warping algorithm (Ashburner 2007) which were subsequently applied to the individual bias-corrected tissue images to produce affine registered GM and WM images. A study-specific DARTEL template was created using these affine registered images from all participants. This longitudinal registration pipeline was then repeated using this study specific DARTEL template to obtain the final modulated images.

The preprocessed structural $\mathrm{T} 1$ and diffusion tensor imaging (DTI) images were then visually inspected for quality. Among the three excluded for image quality issues, two had excessive ghosting effects. As for the third, the DTI acquisition appeared to be incomplete or interrupted; there were missing diffusion directions which resulted in an extremely distorted DTI image. Finally, we used the recently developed HarP hippocampal (Wolf et al. 2017) and younger-older fornix templates (Brown et al. 2017) to extract the hippocampal volumes (HV) as well as fornix FA and MD values. Both ROI templates have been validated in the ADNI sample (Brown et al. 2017; Wolf et al. 2017). The ROI templates were registered to their respective mean study templates prior to the ROI extraction. For each participant, the ROI alignment was visually inspected by overlaying the hippocampus and fornix ROI templates on to their registered T1 and FA images, respectively. Fig. S1 and S2 illustrate the overlays of these ROIs on the mean study images, and the amount of non-zero voxels overlap across volumes. The extracted HVs were then adjusted for total intracranial volume (TIV) using the formula: adjusted $\mathrm{HV}=\mathrm{HV}-\mathrm{b}\left(\mathrm{TIV}-\mathrm{TIV}_{\text {mean }}\right)$, where $\mathrm{b}$ is the regression coefficient for HV against TIV and $\mathrm{TIV}_{\text {mean }}$ is the average TIV of the sample (Buckner et al. 2004).

\section{Statistical analyses}

Auto-regressive cross-lagged models (Anderson and Kida 1982) were implemented to examine the longitudinal associations between fornix WM indices and ADNI-MEM, as well as, between fornix WM indices and HV. In these models, age, sex and education were included as covariates. These models were analyzed via structural equation modeling (SEM) using a robust maximum likelihood estimator. Missing data were assumed to be missing at random; as such, the data was handled with full information maximum likelihood to maximize all available data. These analyses were carried out with the $\mathrm{R}$ package lavaan (Rosseel 2012), in R 3.4.0.

The Comparative Fit Index (CFI) and Standardized Root Mean square Residual (SRMR) were used to assess model fit. The root mean square error of approximation (RMSEA) and chi-square statistics were not used because the relatively small sample size (for an SEM) and degrees of freedom $(\mathrm{df}=1)$ in the current study can significantly bias these estimates (Jackson 2003; Kenny et al. 2015). CFI and SRMR values $\geq .95$ and $\leq$ .10 (Hu and Bentler 1999), respectively, are indicative of acceptable fit. Differences across time in the studied variables were assessed using paired-samples T-tests. Correlations between variables were examined using Pearson correlation coefficients. Statistical significance was set at $p<.05$. 


\section{Results}

\section{Descriptive statistics, bivariate correlations and model fit}

Table 2 presents the correlation matrix of all studied continuous variables. Within each of the cross-sectional time points, fornix FA and MD were significantly associated with $\mathrm{HV}$ and ADNI-MEM. Table 3 presents the descriptive statistics of the longitudinal measures. There were significant changes in the $\mathrm{HV}(t=7.78 ; p<.001)$ as well as fornix FA $(t=5.89 ; p<.001)$ and $\mathrm{MD}(t=4.27 ; p<.001)$ in their expected directions, the decline in ADNI-MEM scores was marginally significant $(t=$ $1.81 ; p<.073)$ the violin plots of these measures are shown in fig. S3 in the supplementary materials. The fit indices of all examined SEM models are presented in Table 4. In general, all models had, at least, acceptable levels of fit.

\section{Cross-lagged analyses of fornix WM and memory}

The results of the cross-lagged analyses between fornix WM indices and ADNI-MEM are presented in Fig. 2. The autoregressive paths in both models were significant; that is, ADNI-MEM scores at baseline significantly predicted its scores at follow-up, and the fornix WM indices at baseline significantly predicted their respective follow-up measures $(\beta \mathrm{s}>.77 ; p \mathrm{~s}<.001)$. Next, ADNI-MEM at baseline significantly predicted fornix FA and MD at follow-up $(p s \leq .046)$ in their expected directions, after controlling for baseline fornix FA and MD respectively. However, the reverse associations were not significant; Fornix FA and MD at baseline did not significantly predict ADNI-MEM at follow-up ( $p \mathrm{~s} \geq .741$ ), after controlling for baseline ADNI-MEM. For the purpose of comparison, a similar cross-lagged model was carried out on $\mathrm{HV}$ and ADNI-MEM; smaller baseline HVs were significantly associated with lower ADNI-MEM scores at follow-up, after controlling for baseline ADNI-MEM scores $(\beta=.14$; $\mathrm{SE}=.05$; $p=.007$; see fig. $\mathrm{S} 4$ in the supplemental materials).

\section{Cross-lagged analyses of HV and fornix measures}

Figure 3 presents the results of the cross-lagged analyses between HV and fornix WM indices. Just as before, all autoregressive paths were highly significant $(\beta \mathrm{s}>.72 ; p \mathrm{~s}$ $<.001)$. HVs at baseline significantly predicted fornix FA and $\mathrm{MD}$ in their expected directions ( $p \mathrm{~s} \leq .002)$, after controlling for baseline fornix FA and MD respectively. The reverse associations were not significant. Fornix FA and MD were not significantly associated with follow-up HVs ( $p s \geq .172$ ), after controlling for baseline HVs.

\section{Sub-analyses}

To test the robustness of our findings, additional sets of crosslagged analyses were carried out within various subgroups. For these analyses, we report only the directional associations that have altered in statistical significance relative to the original analyses (i.e., from significant to non-significant or vice-versa) for brevity. The detailed results of these analyses are presented in the supplementary materials (see table s1 and fig s5-13).

The first set of analyses excluded the participants who were cognitively normal, non-converters, $\mathrm{A} \beta$ - and $\mathrm{APO} \varepsilon 4+$ $\left(\mathrm{N}_{\text {analyzed }}=108\right)$. The results revealed that the ADNI$\mathrm{MEM} \rightarrow$ Fornix MD association had dropped out in statistical significance (from $p=.046$ to $p=.148$ ) (see fig). Furthermore, the fit indices for the Fornix FA $\leftrightarrow \mathrm{HV}$ model had missed the acceptable threshold marginally, resulting in a marginally acceptable fit (see Table S1).

The second set excluded participants who were both $A \beta$ and $\mathrm{APO} \varepsilon 4-$, regardless of their neurocognitive or conversion

Table 2 Correlation matrix of all studied continuous variables

\begin{tabular}{|c|c|c|c|c|c|c|c|c|c|}
\hline Variable & Age baseline & Edu. & Mem. baseline & Mem. follow-up & FA baseline & FA follow-up & MD baseline & MD follow-up & HV baseline \\
\hline \multicolumn{10}{|l|}{ Age $_{\text {baseline }}$} \\
\hline Edu. & -.08 & & & & & & & & \\
\hline Mem.baseline $_{\text {b }}$ & -.11 & .10 & & & & & & & \\
\hline $\mathrm{Mem}_{\text {follow-up }}$ & -.06 & .05 & $.86^{* * * *}$ & & & & & & \\
\hline $\mathrm{FA}_{\text {baseline }}$ & $-.48 * * *$ & .01 & $.23^{*}$ & .18 & & & & & \\
\hline $\mathrm{FA}_{\text {follow-up }}$ & $-.56 * * *$ & -.01 & $.31 * *$ & $.26 * *$ & $.87 * * *$ & & & & \\
\hline $\mathrm{MD}_{\text {baseline }}$ & $.53 * * *$ & .15 & $-.23 *$ & -.19 & $-.89 * * *$ & $-.85 * * *$ & & & \\
\hline $\mathrm{MD}_{\text {follow-up }}$ & $.56 * * *$ & .10 & $-.28 * *$ & $-.24 *$ & $-.82 * * *$ & $-.93 * * *$ & $.93 * * *$ & & \\
\hline $\mathrm{HV}_{\text {baseline }}$ & $-.49 * * *$ & $<.01$ & $.38 * * *$ & $.40 * * *$ & $.59 * * *$ & $.68 * * *$ & $-.59 * * *$ & $-.65 * * *$ & \\
\hline $\mathrm{HV}_{\text {follow-up }}$ & $-.49 * * *$ & .03 & $.43 * * *$ & $.43 * * *$ & $.60 * * *$ & $.71 * * *$ & $-.60 * * *$ & $-.67 * * *$ & $.93 * * *$ \\
\hline
\end{tabular}

Edu, Education in years; Mem, memory composite scores (ADNI-MEM); FA, Fractional Anisotropy of fornix; MD, Mean Diffusivity of fornix; HV, Hippocampal volume (adjusted for intracranial volume). $* p<.05 ; * * p<.01 ; * * *<<.001$ 
Table 3 Descriptive statistics of longitudinal measures

\begin{tabular}{|c|c|c|c|c|c|c|c|c|}
\hline & \multicolumn{2}{|c|}{ ADNI-MEM } & \multicolumn{3}{|l|}{ Fornix } & \multicolumn{3}{|c|}{ T1 Structural } \\
\hline & Valid N & $\mathrm{M}(\mathrm{SD})$ & Valid N & $\begin{array}{l}\text { FA } \\
\mathrm{M}(\mathrm{SD})\end{array}$ & $\begin{array}{l}\mathrm{MD}\left(10^{-3} \mathrm{~mm}^{2} / \mathrm{s}\right) \\
\mathrm{M}(\mathrm{SD})\end{array}$ & Valid N & $\begin{array}{l}\mathrm{HV}\left(\mathrm{cm}^{3}\right) \\
\mathrm{M}(\mathrm{SD})\end{array}$ & $\begin{array}{l}\mathrm{TIV}\left(\mathrm{cm}^{3}\right) \\
\mathrm{M}(\mathrm{SD})\end{array}$ \\
\hline Baseline & 115 & $.417(.693)$ & 98 & $.350(.048)$ & $1.399(.202)$ & 112 & $7.243(1.031)$ & $1477(131)$ \\
\hline Follow-up & 115 & $.339(.897)$ & 98 & $.334(.053)$ & $1.433(.216)$ & 112 & $6.926(1.177)$ & $1479(135)$ \\
\hline Follow-up - baseline & 115 & $-.078(.465)$ & 98 & $-.016(.026)$ & $.034(.079)$ & 112 & $-.316(.145)$ & $2.521(26.3)$ \\
\hline
\end{tabular}

ADNI-MEM, memory composite scores; FA, Fractional Anisotropy; MD, Mean Diffusivity; HV, Hippocampal volume (adjusted for intracranial volume); TIV, intracranial volume; M, Mean; SD, Standard Deviation

status $\left(\mathrm{N}_{\text {analyzed }}=94\right)$. Similarly, the ADNI-MEM $\rightarrow$ Fornix MD association had dropped out in statistical significance, though very marginally (from $\mathrm{p}=.046$ to $p=.058$ ) (see fig. S7).

To further examine the study's hypotheses among individuals with significant evidence of $\mathrm{AD}$ pathophysiological process, we identified 68 participants among all $\mathrm{A} \beta+$ participants, who were CSF tau positive (p-tau $\geq 23 \mathrm{pg} / \mathrm{ml}$ (Shaw et al. 2009)) and entered them into the same analyses. In these results, the previously non-significant Fornix FA $\rightarrow$ HV association had become statistically significant (from $p=.193$ to $p=.025)$ (see fig. S10).

Relatedly, we also carried out the analyses among participants have little or no evidence of the AD pathophysiological process. These are participants who were $A \beta$ - regardless of their neurocognitive, conversion or APO $\varepsilon 4$ status $\left(\mathrm{N}_{\text {analyzed }}=40\right)$. There were major differences between the results of these analyses and those of the original. The ADNI-MEM $\rightarrow$ Fornix MD, ADNI-MEM $\rightarrow$ Fornix FA and HV $\rightarrow$ Fornix MD associations were no longer statistically significant ( $\mathrm{ps} \geq .091$ ), whereas the previously nonsignificant association of Fornix MD $\rightarrow \mathrm{HV}$ had become statistically significant ( $p=.172$ to $p=.027$ ) (see fig. S11 and S12).

Finally, we controlled for participants' baseline neurocognitive status (i.e., cognitive normal or MCI due to AD) in cross-lagged analyses of Fornix FA $\leftrightarrow \mathrm{HV}$ and Fornix $\mathrm{MD} \leftrightarrow \mathrm{HV}$, by including them as covariates. No participants

Table 4 Fit indices of all examined SEM models

\begin{tabular}{lcc}
\hline Model & CFI & SRMR \\
\hline Fornix FA $\leftrightarrow$ ADNI-MEM & .993 & .039 \\
Fornix MD $\leftrightarrow$ ADNI-MEM & .994 & .039 \\
Fornix FA $\leftrightarrow$ HV & .950 & .095 \\
Fornix MD $\leftrightarrow$ HV & .960 & .093 \\
\hline
\end{tabular}

CFI, Comparative Fit Index; SRMR, Standardized Root Mean Square residual; FA, Fractional Anisotropy; MD, Mean Diffusivity; HV, Hippocampal volume (adjusted for intracranial volume); ADNI-MEM, memory composite scores were excluded in this set of analyses. (see fig. S13). Relative to the original analyses, the statistical significance for all directional associations were similar (see fig. S13). We did not repeat the analyses of Fornix FA $\leftrightarrow$ ADNI-MEM and Fornix $\mathrm{MD} \leftrightarrow \mathrm{ADNI}-\mathrm{MEM}$ to control for neurocognitive status since such diagnoses were largely contingent on the memory scores.

\section{Discussion}

We have demonstrated the directional relationships between the microstructure of the fornix, verbal memory, and hippocampal volume in the context of AD progression. These findings do not support our bidirectional hypothesis of disrupted fornix microstructure and memory impairments. While impaired verbal memory and smaller hippocampal volumes at baseline significantly predicted worse fornix microstructure two years later, the reverse direction of associations were not found to be significant. Baseline fornix microstructure was not significantly associated with subsequent memory impairment or hippocampal volumes. Our findings did however support the second hypothesis on hippocampal atrophy predicting the microstructure of the fornix at follow-up. It should be noted that these finding are unlikely to be driven by baseline differences in memory, fornix microstructure or $\mathrm{HV}$, as result of diagnosis status or other factors, since these baseline variables are already controlled for in our autoregressive models.

We carried out additional analyses to examine if such associations hold within various subgroups. The results of these analyses were largely similar to our main findings, with some interesting exceptions. First, among the group of individuals with significant $\mathrm{AD}$ pathophysiology, there was a significant bidirectional relationship between fornix FA and HV. Fornix FA predicted subsequent $\mathrm{HV}$ and vice versa. These findings suggest that the axial or radial degeneration of fornix WM tracts (but not both, hence impacting FA more than MD), tends to be tightly coupled with hippocampal atrophy. Next, among participants with little or no AD pathophysiology, there were no significant associations between fornix FA/MD and memory in any direction. Relative to the main analyses, the directional association between fornix MD and HV had reversed, while that of 
fornix FA and HV had remained the same. These findings suggest the combined axial and radial degeneration (i.e., MD) of fornix WM fibers preceded hippocampal atrophy in the early stages. Then, following significant hippocampal atrophy, the fornix WM fiber tracts continued to degenerate, however, only in an axial or radial manner to influence FA more so than MD. Nevertheless, regardless of the type of fornix WM degeneration, the changes fornix microstructure did not appear to be related to memory decline. The findings of these two sets of analyses taken together would very roughly suggest that AD pathophysiology accelerates hippocampal atrophy and memory decline more so than the deterioration of fornix microstructure, especially in that of MD. An alternative or perhaps less plausible interpretation would be that $\mathrm{AD}$ pathophysiology delays alterations in fornix microstructure, until significant memory decline and hippocampal atrophy have occurred. Given the small sample sizes associated with these analyses, these interpretations are rudimentary at best and should be clarified with larger sampled studies.

Our findings from the main analyses are consistent with Fletcher et al.'s (2013) observations that fornix FA do not significantly predict subsequent conversion to $\mathrm{aMCI}$ or $\mathrm{AD}$ ).
In another similar study, Zhuang et al.'s (2012) whole brain tract-based spatial statistics revealed that aMCI converters had significantly lower fornix FA at baseline. Nevertheless, it was not predictive of subsequent verbal episodic memory in their multiple regression models. Such conflicting findings, within a study, alluded to the idea that fornix FA may not be a robust predictor of subsequent memory decline in general. Likewise, although Mielke et al. (2012) documented a significant association between baseline fornix FA and subsequent memory impairment, similar to the present study, the magnitude of such association was reportedly very small (standardized coefficient $=.01$ ) compared to that of HV and subsequent memory impairment. Our results instead provide support for the reverse association - memory impairments significantly predict subsequent disruption in fornix microstructure. This is consistent with Nowrangi et al.'s (2013) report of individuals with aMCI or $\mathrm{AD}$, relative to healthy controls, presenting with significantly increased fornix MD over time. Although Teipel et al. (2014) did not report a significant fornix FA decline in their follow-up of participants with aMCI relative to healthy controls, the absolute decline in FA in the former group was approximately four times larger than the latter. Given the small

Fig. 2 Robust Maximum

Fornix FA $\leftrightarrow$ ADNI-MEM

likelihood estimation of the crosslagged effects between extracted regions of interest and ADNI-

MEM, with age, sex and education included as covariates. Straight lines represent regression paths. Curve line represents residual covariance.

FA = Fractional Anisotropy;

$\mathrm{MD}=$ Mean Diffusivity;

$\beta=$ standardized coefficients; SE

$=$ Standard Error

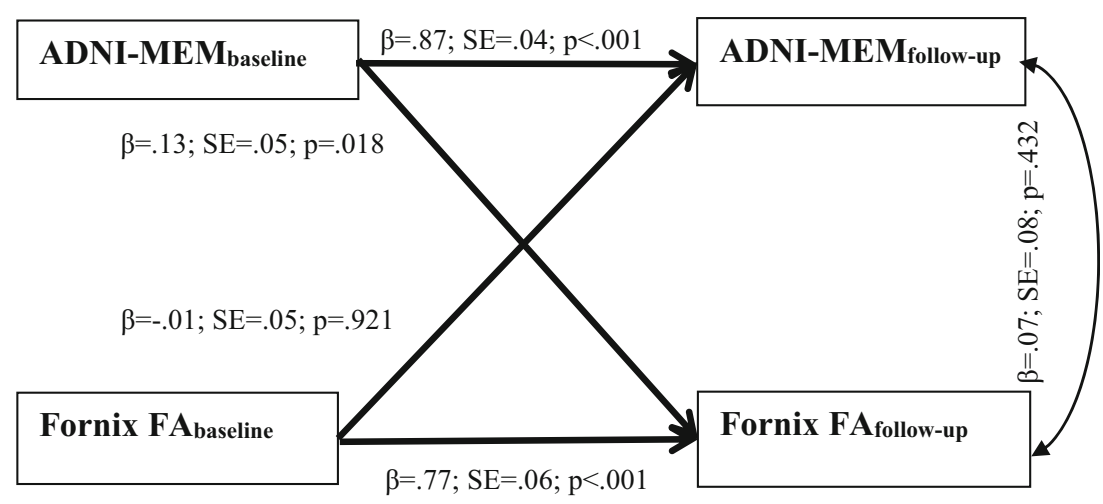

Fornix MD $\leftrightarrow$ ADNI-MEM

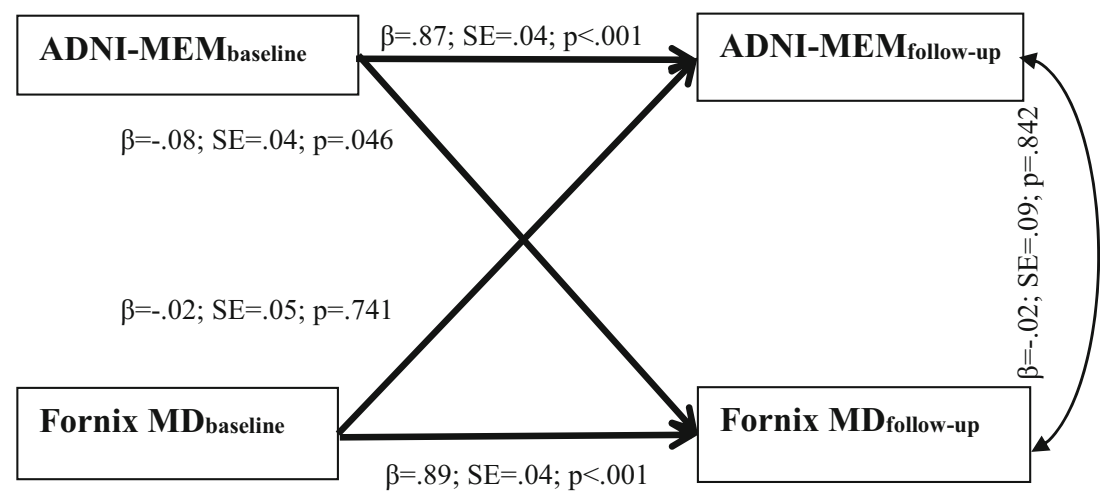


sample size, it is possible that their study was underpowered to detect a significant time by group interaction. Overall, the accumulated evidence suggests memory impairment precedes changes in fornix microstructure.

In relation to the second hypothesis, Fletcher et al. (2013) previously reported cross-sectional associations between $\mathrm{HV}$ and fornix microstructure. Our novel longitudinal findings go one step further to illustrate the directional nature of such associations. We found that smaller HVs were associated with worse fornix microstructure at follow-up, however, the reverse association (i.e. fornix microstructure predicting subsequent HVs) was not significant. Relatedly, a previous longitudinal study illustrated a similar sequential relationship between hippocampal atrophy and WM pathology with baseline hippocampal volumes predicting subsequent WM atrophy in the cingulum and uncinate fasciculus (Villain et al. 2010). These findings converge to suggest WM pathology is likely to appear in the late prodromal stage of AD where there has already been a noticeable decline in memory and HV.

\section{Insights into the mechanisms of AD progression in humans}

Our findings advance the current understanding of the pathophysiological processes involved in the early prodromal stages of AD. The observation of hippocampal atrophy predicting subsequent disruption in fornix microstructure is consistent with Kantarci's (2014) proposal of hippocampal neuronal death preceding subsequent degeneration of fornix WM fiber tracts. Along with the fact that hippocampal atrophy predicted subsequent memory impairment, these findings provide support for the amyloid cascade hypothesis. The possible notion that AD pathophysiology accelerates hippocampal atrophy and memory decline more so than the deterioration of fornix microstructure, as alluded to above, would also be consistent with such a hypothesis. On the other hand, given that fornix microstructure did not significantly predict subsequent memory impairment, such memory impairment is unlikely to be associated with a WM-related pathology, at least in the fornix. Taken together, these findings cast doubt on the
Fig. 3 Robust Maximum likelihood estimation of the crosslagged effects between extracted regions of interest and hippocampal volume, with age, sex and education included as covariates. Straight lines represent regression paths. Curve line represents residual covariance. $\mathrm{FA}=$ Fractional Anisotropy; $\mathrm{MD}=$ Mean Diffusivity; HV = Hippocampal volume (adjusted for intracranial volume); $\beta=$ standardized coefficients; $\mathrm{SE}=$ Standard Error
Fornix FA $\leftrightarrow$ HV

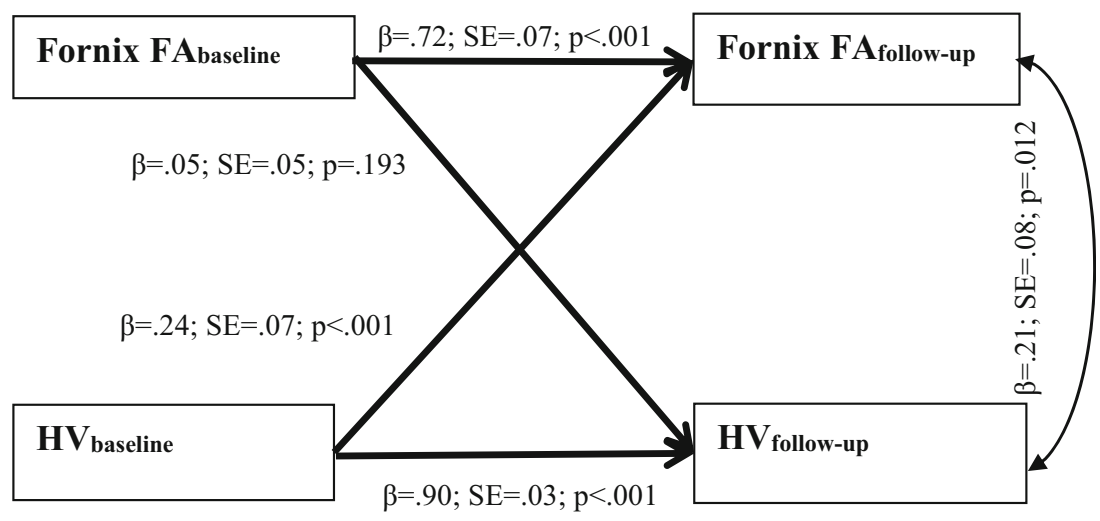

Fornix MD $\leftrightarrow$ HV

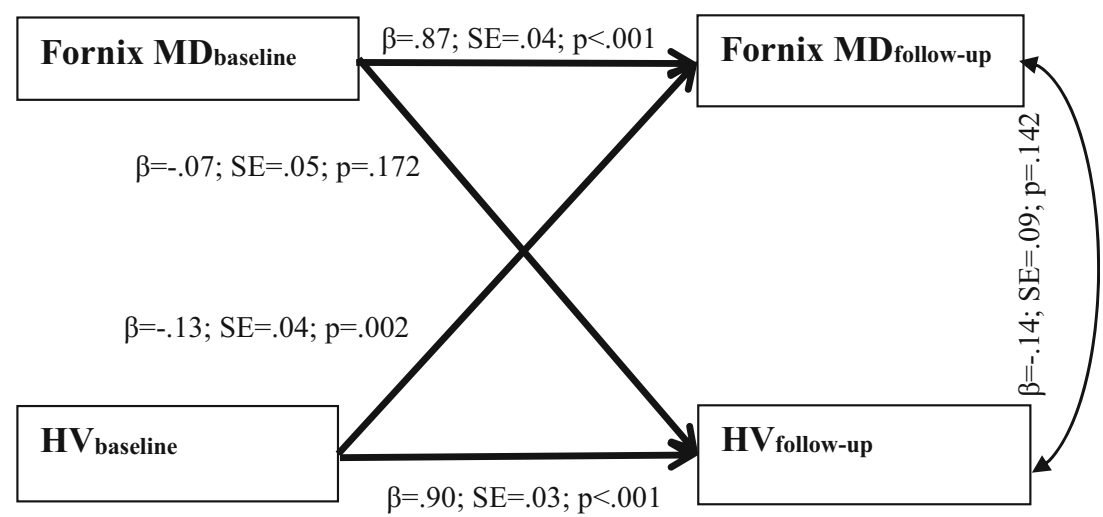


possibility of neuroinflammatory processes implicating the fornix in the pathogenesis of AD. Nevertheless, we cannot rule out the possibility of neuroinflammatory processes acting on other WM regions commonly observed to be altered in prodromal stages of $\mathrm{AD}$ apart from the fornix, such as the uncinate fasciculus and parahippocampal cingulum (Yu et al. 2017). Future longitudinal research may consider employing a whole-brain approach to investigate if certain memoryassociated WM microstructural alterations in the brain are independent, or even precede, hippocampal atrophy to examine such a possibility.

\section{Significant clinical implications}

Results of our study provide important information in regard to the use of neural markers in the context of prodromal AD. Previous cross-sectional studies have frequently observed altered fornix microstructure in the prodromal stages of $\mathrm{AD}$, and on the basis of this cross-sectional evidence some have concluded that this pathology represents an early marker of $\mathrm{AD}$ (for a review see Nowrangi and Rosenberg (2015)). Whilst this conclusion is not wrong, our findings suggest it is not entirely correct. Our observation of fornix microstructure being predicted by preceding hippocampal atrophy and memory impairment suggests that fornix microstructural alterations do not occur early enough in the course of prodromal $\mathrm{AD}$; these alterations may indicate an individual to be in a late prodromal stage of $\mathrm{AD}$. As such, if fornix microstructure was used as an early marker in the assessment of $\mathrm{AD}$, one would have incorrectly identified individuals in the late prodromal stage as belonging to the early stage. The implication of this inaccurate identification cannot be understated especially given that the best course of treatment in $\mathrm{AD}$ largely depends on the stage of the disease (Anand et al. 2014). In tracking the early progression of $A D$, hippocampal volumetric measurements, relative to fornix microstructural indices, are perhaps the more useful noninvasive neural markers given their value in predicting future memory decline. Next, the current study together with a previous work (Villain et al. 2010) demonstrates the sequential nature of GM and WM pathology. Based on this information, one could chart the progression of AD more confidently via the use of multiple GM and WM markers to better inform the optimal course of treatment.

\section{Limitations}

In the current study, we did not carry out free water elimination (Pasternak et al. 2009), as such the fornix WM indices may have been susceptible to CSF contamination, especially given its intraventricular location. Nevertheless, we have attempted to minimize such partial volume effects by only including voxels in the thresholded mean FA skeleton into the fornix ROI. Next, our interpretation of the study's findings on $\mathrm{AD}$ related pathophysiological process is limited by the fact that not all of these participants would eventually develop $\mathrm{AD}$ in their lifetime. Strengths of the current study include a relatively large sample size and consistency in the effects relating to both DTI indices for the main analyses. The latter alluded to the robustness of our results given that previous studies in this area have generally found significant effects in one DTI measure, but not the other.

\section{Conclusions}

The current study sought to evaluate the utility of fornix microstructure as an early neural marker of AD by examining its longitudinal relationship with memory impairment and hippocampal atrophy. We found that memory impairment and HVs significantly predicted subsequent disruption in fornix microstructure. However, fornix microstructure was not significantly associated with subsequent memory impairment and HVs. These results suggest disruptions in fornix microstructure are likely to occur in the late prodromal AD stage, where significant memory impairment and hippocampal atrophy has already occurred. These longitudinal findings are crucial in refining the use of these neural markers in the early detection of $\mathrm{AD}$ and advancing our understanding of development of AD.

Acknowledgements This work is supported by funding from The University of Hong Kong May Endowed Professorship, the KKHo International Charitable Foundation and, the Science and Technology Program of Guangdong (Ref: 2018B030334001). Data collection and sharing for this project was funded by the ADNI (National Institutes of Health Grant U01 AG024904) and DOD ADNI (Department of Defense award number W81XWH-12-2-0012). ADNI is funded by the National Institute on Aging, the National Institute of Biomedical Imaging and Bioengineering, and through generous contributions from the following: AbbVie, Alzheimer's Association; Alzheimer's Drug Discovery Foundation; Araclon Biotech; BioClinica, Inc.; Biogen; Bristol-Myers Squibb Company; CereSpir, Inc.; Cogstate; Eisai Inc.; Elan Pharmaceuticals, Inc.; Eli Lilly and Company; EuroImmun; F. Hoffmann-La Roche Ltd. and its affiliated company Genentech, Inc.; Fujirebio; GE Healthcare; IXICO Ltd.; Janssen Alzheimer Immunotherapy Research \& Development, LLC.; Johnson \& Johnson Pharmaceutical Research \& Development LLC.; Lumosity; Lundbeck; Merck \& Co., Inc.; Meso Scale Diagnostics, LLC.; NeuroRx Research; Neurotrack Technologies; Novartis Pharmaceuticals Corporation; Pfizer Inc.; Piramal Imaging; Servier; Takeda Pharmaceutical Company; and Transition Therapeutics. The Canadian Institutes of Health Research is providing funds to support ADNI clinical sites in Canada. Private sector contributions are facilitated by the Foundation for the National Institutes of Health (www.fnih.org). The grantee organization is the Northern California Institute for Research and Education, and the study is coordinated by the Alzheimer's Therapeutic Research Institute at the University of Southern California. ADNI data are disseminated by the Laboratory for Neuro Imaging at the University of Southern California. 
Compliance with ethical standards Ethical approval for the ADNI study was obtained by the ADNI investigators.

Conflict of interest The authors declare no conflict of interest.

Informed consent All participants gave informed consent before participating in the study.

Open Access This article is distributed under the terms of the Creative Commons Attribution 4.0 International License (http:// creativecommons.org/licenses/by/4.0/), which permits unrestricted use, distribution, and reproduction in any medium, provided you give appropriate credit to the original author(s) and the source, provide a link to the Creative Commons license, and indicate if changes were made.

\section{References}

Anand, R., Gill, K. D., \& Mahdi, A. A. (2014). Therapeutics of Alzheimer's disease: Past, present and future. Neuropharmacology, $76,27-50$.

Anderson, T. N., \& Kida, T. E. (1982). The cross-lagged research approach: Description and illustration. Journal of Accounting Research, 20(2), 403-414. https://doi.org/10.2307/2490748.

Ashburner, J. (2007). A fast diffeomorphic image registration algorithm. Neuroimage, 38(1), 95-113.

Brown, C. A., Johnson, N. F., Anderson-Mooney, A. J., Jicha, G. A., Shaw, L. M., Trojanowski, J. Q., et al. (2017). Development, validation and application of a new fornix template for studies of aging and preclinical Alzheimer's disease. NeuroImage: Clinical, 13(Supplement C), 106-115. https://doi.org/10.1016/j.nicl.2016. 11.024

Buckner, R. L., Head, D., Parker, J., Fotenos, A. F., Marcus, D., Morris, J. C., \& Snyder, A. Z. (2004). A unified approach for morphometric and functional data analysis in young, old, and demented adults using automated atlas-based head size normalization: Reliability and validation against manual measurement of total intracranial volume. NeuroImage, 23(2), 724-738. https://doi.org/10.1016/j. neuroimage.2004.06.018.

Caso, F., Agosta, F., \& Filippi, M. (2016). Insights into white matter damage in Alzheimer's disease: From postmortem to in vivo diffusion tensor MRI studies. Neuro-Degenerative Diseases, 16(1-2), 26-33. https://doi.org/10.1159/000441422.

Crane, P. K., Carle, A., Gibbons, L. E., Insel, P., Mackin, R. S., Gross, A., et al. (2012). Development and assessment of a composite score for memory in the Alzheimer's Disease Neuroimaging Initiative (ADNI). Brain Imaging and Behavior, 6(4), 502-516. https://doi. org/10.1007/s11682-012-9186-z.

Fletcher, E., Raman, M., Huebner, P., Liu, A., Mungas, D., Carmichael, O., \& DeCarli, C. (2013). Loss of fornix white matter volume as a predictor of cognitive impairment in cognitively normal elderly individuals. JAMA Neurology, 70(11), 1389-1395. https://doi.org/10. 1001/jamaneurol.2013.3263.

Hardy, J., \& Selkoe, D. J. (2002). The amyloid hypothesis of Alzheimer's disease: Progress and problems on the road to therapeutics. Science, 297(5580), 353-356.

Heppner, F. L., Ransohoff, R. M., \& Becher, B. (2015). Immune attack: The role of inflammation in Alzheimer disease. Nature Reviews Neuroscience, 16(6), 358-372. https://doi.org/10.1038/nrn3880.

Hu, L., \& Bentler, P. M. (1999). Cutoff criteria for fit indexes in covariance structure analysis: Conventional criteria versus new alternatives. Structural Equation Modeling: A Multidisciplinary Journal, 6(1), 1-55. https://doi.org/10.1080/10705519909540118.
Jackson, D. L. (2003). Revisiting sample size and number of parameter estimates: Some support for the N:Q hypothesis. Structural Equation Modeling: A Multidisciplinary Journal, 10(1), 128-141. https://doi.org/10.1207/S15328007SEM1001_6.

Kantarci, K. (2014). Fractional anisotropy of the fornix and hippocampal atrophy in Alzheimer's disease. Frontiers in Aging. Neuroscience, 6(OCT), 1-4. https://doi.org/10.3389/fnagi.2014.00316.

Keihaninejad, S., Zhang, H., Ryan, N. S., Malone, I. B., Modat, M., Cardoso, M. J., et al. (2013). An unbiased longitudinal analysis framework for tracking white matter changes using diffusion tensor imaging with application to Alzheimer's disease. Neuroimage, 72, 153-163. https://doi.org/10.1016/j.neuroimage.2013.01.044.

Kenny, D. A., Kaniskan, B., \& McCoach, D. B. (2015). The performance of RMSEA in models with small degrees of freedom. Sociological Methods \& Research, 44(3), 486-507. https://doi.org/10.1177/ 0049124114543236.

Mielke, M. M., Okonkwo, O. C., Oishi, K., Mori, S., Tighe, S., Miller, M. I., et al. (2012). Fornix integrity and hippocampal volume predict memory decline and progression to Alzheimer's disease. Alzheimer's and Dementia, 8(2), 105-113. https://doi.org/10.1016/ j.jalz.2011.05.2416.

Nowrangi, M. A., \& Rosenberg, P. B. (2015). The fornix in mild cognitive impairment and Alzheimer's disease. Frontiers in Aging Neuroscience. http://journal.frontiersin.org/article/10.3389/fnagi. 2015.00001.

Nowrangi, M. A., Lyketsos, C. G., Leoutsakos, J.-M. S., Oishi, K., Albert, M., Mori, S., \& Mielke, M. M. (2013). Longitudinal, region-specific course of diffusion tensor imaging measures in mild cognitive impairment and Alzheimer's disease. Alzheimer's \& Dementia, 9(5), 519-528.

Oishi, K., \& Lyketsos, C. G. (2014). Alzheimer's disease and the fornix. Frontiers in Aging Neuroscience, 6(SEP), 1-9. https://doi.org/10. 3389/fnagi.2014.00241.

Pasternak, O., Sochen, N., Gur, Y., Intrator, N., \& Assaf, Y. (2009). Free water elimination and mapping from diffusion MRI. Magnetic Resonance in Medicine, 62(3), 717-730. https://doi.org/10.1002/ mrm.22055.

Rosseel, Y. (2012). lavaan: An R Package for Structural Equation Modeling. Journal of Statistical Software; Vol 1, Issue 2 (2012). https://doi.org/10.18637/jss.v048.i02

Shaw, L. M., Vanderstichele, H., Knapik-Czajka, M., Clark, C. M., Aisen, P. S., Petersen, R. C., et al. (2009). Cerebrospinal fluid biomarker signature in alzheimer's disease neuroimaging initiative subjects. Annals of Neurology, 65(4), 403-413. https://doi.org/10.1002/ana. 21610.

Sperling, R. A., Aisen, P. S., Beckett, L. A., Bennett, D. A., Craft, S., Fagan, A. M., et al. (2011). Toward defining the preclinical stages of Alzheimer's disease: Recommendations from the National Institute on Aging-Alzheimer's association workgroups on diagnostic guidelines for Alzheimer's disease. Alzheimer's \& dementia : the journal of the Alzheimer's Association, 7(3), 280-292. https://doi.org/10. 1016/j.jalz.2011.03.003.

Teipel, S. J., Meindl, T., Wagner, M., Stieltjes, B., Reuter, S., Hauenstein, K. H., et al. (2010). Longitudinal changes in fiber tract integrity in healthy aging and mild cognitive impairment: A DTI follow-up study. Journal of Alzheimer's Disease, 22(2), 507-522. https://doi. org/10.3233/JAD-2010-100234.

Teipel, S. J., Grothe, M. J., Filippi, M., Fellgiebel, A., Dyrba, M., Frisoni, G. B., et al. (2014). Fractional anisotropy changes in Alzheimer's disease depend on the underlying fiber tract architecture: A multiparametric DTI study using joint independent component analysis. Journal of Alzheimer's Disease, 41(1), 69-83.

Villain, N., Fouquet, M., Baron, J.-C., Mézenge, F., Landeau, B., de La Sayette, V., et al. (2010). Sequential relationships between grey matter and white matter atrophy and brain metabolic abnormalities in early Alzheimer's disease. Brain, 133(11), 3301-3314. 
Wolf, D., Bocchetta, M., Boccardi, M., Bocchetta, M., Preboske, G. M., Boccardi, M., \& Grothe, M. J. (2017). Reference standard space hippocampus labels according to the European Alzheimer's disease consortium-Alzheimer's Disease Neuroimaging Initiative harmonized protocol: Utility in automated volumetry. Alzheimer's and Dementia, 13(8), 893-902. https://doi.org/10.1016/j.jalz.2017.01. 009.

Yu, J., Lam, C. L. M., \& Lee, T. M. C. (2017). White matter microstructural abnormalities in amnestic mild cognitive impairment: A metaanalysis of whole-brain and ROI-based studies. Neuroscience and
Biobehavioral Reviews, 83(June), 405-416. https://doi.org/10.1016/ j.neubiorev.2017.10.026.

Zhuang, L., Wen, W., Trollor, J. N., Kochan, N. A., Reppermund, S., Brodaty, H., \& Sachdev, P. (2012). Abnormalities of the fornix in mild cognitive impairment are related to episodic memory loss. Journal of Alzheimer's Disease, 29(3), 629-639.

Publisher's note Springer Nature remains neutral with regard to jurisdictional claims in published maps and institutional affiliations. 